\title{
Cognitive Impairment in Schizophrenia: Current Perspective
}

\author{
Aich TK ${ }^{1}$, Mahato $\mathbf{A}^{2}$, Subedi $\mathbf{S}^{3}$ \\ 1.Associate Professor and Head, Department of Psychiatry, BRD Medical College, Gorakhpur, UP, India \\ 2. Associate Professor in Clinical Psychology \& Principal, Sweekaar academy of Rehabilitation \\ Sciences, Secunderabad, AP, India 3. Associate Professor, Department of Psychiatry, UCMS, \\ Bhairahawa, Nepal
}

E-mail *Corresponding author: tapas_dr@yahoo.co.in

\begin{abstract}
Impairments in a variety of cognitive functions are found in patients with schizophrenia. These impairments affect a wide array of different cognitive abilities and are often of moderate to severe degree. Cognitive impairments appear to present across lifespan, detectable at the time of first episode of illness, probably predate the illness and manifest a generally stable course over time.

Though cognitive impairment does not form a part of diagnostic criteria, it has been included in DSM-V and proposed to be included in ICD-11 as a schizophrenia course specifier. This review attempts to provide a broad overview of the domains, onset, severity and course of cognitive impairments in schizophrenia, with a focus on functional relevance and treatment possibilities. There is strong evidence for a relationship between cognitive impairment and vocational and functional impairment in individuals with schizophrenia.
\end{abstract}

Keywords: Schizophrenia, Cognitive Deficits, Functional Relevance, Cognitive Remediation

\section{INTRODUCTION}

Cognitive impairment and diagnosis of schizophrenia

Traditionally, significant cognitive impairment was thought to be evident in elderly deteriorated patients with schizophrenia. Current evidence, however, is challenging this view. It is becoming evident that marked cognitive impairment is the norm and often predates the illness. However, cognitive impairment is still not a formal part of the current diagnostic criteria for schizophrenia. But experts have increasingly expressed the idea that neurocognitive impairment is a core feature of the illness.

DSM-V mentioned about the characteristic symptoms of schizophrenia involving a range of cognitive, behavioral, and emotional dysfunctions. Cognitive deficits include decrements in declarative memory, working memory, language function, and other executive functions, as well as slower processing speed. In addition to the five symptom domain areas identified in the diagnostic criteria, DSM-V highlighted the need for assessment of cognition, depression and mania in distinguishing various schizophrenia spectrum and other psychotic disorders ${ }^{1}$.

The ICD-11 working group suggested to replace the classical schizophrenia subtypes by six symptom specifiers (positive, negative, depressive, manic, and psychomotor symptoms, and cognitive impairment), each to be coded separately. Cognitive impairments as part of the symptom specifiers will be introduced to the spectrum of schizophrenia symptoms given their high importance for the clinical course ${ }^{2}$. 
Clinical Vignette: A young lady in her early thirties brought to psychiatry OPD by her daughter who was in her mid-teens. She was diagnosed to have schizophrenia, presenting with predominantly catatonic symptoms. She responded very well to treatment with total return to her normal premorbid state. Subsequent episodes were marked by symptom fluctuation, from "paranoid" presentation to "undifferentiated" to "negative", "chronic", apathetic" state. With passage of time symptoms became more severe, longer, with lesser interepisode normal interval and more and more treatment resistance state. She needed admission, ECT, medication changed to Clozapine and combination of drugs were tried. There was obvious deterioration in cognitive performance over the period of time.

Many senior psychiatrists, who are in this field for more than 15-20 years, have come across patients like this in their routine clinical practice. Looking back into her clinical course many questions creps into one's mind: Cognitive decline that we see in our patients is how much important in diagnosis of schizophrenia? Cognitive impairment is the cause or the consequence of schizophrenia? Does drugs has a role to play in this cognitive impairment? How best we can diagnose cognitive impairment in schizophrenic patients? Can this cognitive impairment be treated or its progress can be arrested? Present article will try to shed some light in above and few other questions related to cognitive deficits in schizophrenic patient population.

Three era of neuro-cognitive studies in schizophrenia

Neuro-cognitive studies in schizophrenia has passed through three phases during the past several decades. Early studies were usually done before 1980s.

During early studies efforts were to demonstrate schizophrenia as a brain disease. This phase was supported primarily through the use of neuroimaging techniques such as computerized tomography (CT), which consistently showed that patients had diffuse nonspecific abnormalities such as prominent sulci or ventricular enlargement ${ }^{3,4}$.
Middle period saw the studies based on the traditions of neurology and neuropsychology. Researchers had the facilities of advance neuroimaging techniques like MRI and they attempted to localize the anatomic abnormalities and relate specific manifestations of schizophrenia to specific brain regions ${ }^{5}$.

Recent studies draws on traditions of cognitive psychology, models of distributed parallel processing, and the study of neural circuitry ${ }^{6}$. The emphasis of this phase is an attempt to understand schizophrenia as an abnormality in fundamental cognitive processes and distributed circuits. The emphasis has shifted to develop an integrative model of schizophrenia ${ }^{7}$.

\section{Cognitive deficits in Schizophrenia: to what extent?}

Up to $98 \%$ of patients with schizophrenia perform poorly on cognitive tests than would be predicted by their parents' education level. In several cognitive domains, the average cognitive impairment in schizophrenia can reach two standard deviations below the healthy control mean ${ }^{8,9}$. Comparisons of monozygotic twins discordant for schizophrenia suggest that almost all affected twins perform worse than their unaffected twin on cognitive tests ${ }^{10}$. Therefore, it is likely that almost all patients with schizophrenia are functioning below the level that would be expected in the absence of the illness.

Is it possible to be schizophrenic yet neuropsychologically normal? Palmer et al (1997) explored the possible answers to this question by giving a comprehensive neuropsychological battery to 171 out-patients with schizophrenia and compared them with 63 healthy controls ${ }^{11}$. Only $27 \%$ of the patients with schizophrenia were classified as neuropsychologically 'normal'. This indicates that significant cognitive impairment in schizophrenia is, in fact, the norm. However, this study also highlights the fact that a proportion of patients with schizophrenia appears to remain neuropsychologically intact! 
Does the cognitive impairment pre-date the illness?

Works completed in UK, USA and Sweden suggested that children who went on to develop schizophrenia as adults differed from the general population in a wide range of cognitive and behavioural domains ${ }^{12-14}$. In Israel, a study of all adolescents between the ages of 16 and 17 years suggested that cognitive functions are significantly impaired in those adolescents who are later hospitalized for schizophrenia ${ }^{15}$. North American Prodromal Longitudinal Study (NAPLS) consortium also indicate poorer scores for those high-risk children who would develop schizophrenia in later life ${ }^{16}$. Prospective studies have suggested that cognitive impairment is manifest in individuals who are identified as being at "ultra-high risk" for schizophrenia by virtue of their family history of schizophrenia and/or had evidence of prodromal symptoms of schizophrenia ${ }^{17}$.

Is the cognitive impairment in schizophrenia caused by medication?

Most neuropsychological studies in schizophrenia have been conducted on medicated patients. Several studies have appeared over the years which have indicated marked and severe cognitive impairment in patients who have been taken off their medication, or in patients who have never been prescribed any neuroleptic or anticholinergic medication $^{18,19}$. Thus, although drugs may make a contribution, they do not account for the cognitive impairment that is observed in schizophrenia.

\section{Is the cognitive impairment progressive?}

The concept of 'dementia precox' introduced by Kraepelin focussed on the cognitive and functional deficits with a progressive cognitive decline likened the condition to a dementic illness with an earlier age of onset ${ }^{20}$. One of the outstanding issues is whether or not the cognitive impairment observed in patients with schizophrenia declines over time. There are opposing views on this subject ${ }^{21}$. Russell et al (1997) followed up patients who had received an intelligence test as a child and later on developed schizophrenia. Their intelligence were re-tested some 19 years later. There were no differences between the child and adult IQs. Thus, they concluded that intellectual decline in schizophrenia was a nothing more than a 'myth'21.

Rund (1998) has reviewed a number of longitudinal studies and concluded that the cognitive deficits appear to be relatively stable in schizophrenia, consistent with a static encephalopathy rather than a degenerative process $^{22}$. However, most clinicians are able to identify individual patients who appear to have declined significantly cognitively since the onset of their illness. It may be that subgroups of patients show a particularly poor cognitive outcome.

\section{Domains of cognitive functions and methods of assessment for the same}

A large variety of cognitive function tests are available and are utilized in schizophrenia research over decades ${ }^{23}$. MATRICS consensus cognitive battery (MCCB) includes ten tests of cognition in seven domains. The MCCB has been established as the gold standard for cognitive research in schizophrenia. Domains of cognitive tests done are speed of processing, attention and vigilance, working memory, verbal and visual learning, reasoning and problem solving and social cognition. For assessing above cognitive domains we have test batteries like Category fluency, BACS symbol coding and Trail making A tests, Continuous performance test, Letter-number span and WMS-III spatial span tests, Hopkins verbal learning test- $R$, and 'Brief visuospatial memory test-R'. MCCB uses 'NAB mazes' test for Reasoning and problem solving and 'MSCEIT managing emotions' test for Social cognition. These battery of tests were chosen on the basis that these tests were in the key domains of cognition in schizophrenia, had excellent psychometric properties, had relations to functional outcomes, were practical for use in clinical trials, and were not burdensome for patients ${ }^{24}$.

Neural localization of cognitive impairment in schizophrenia?

Various authors have concluded that schizophrenia is characterised by cognitive test 
profiles indicative of dysfunction of the frontal lobe, temporal lobe, left or right hemisphere, basal ganglia, etc. ${ }^{25}$. This lack of consensus may reflect the heterogeneity of schizophrenia, and may also be a result of the relatively poor localising ability of many standard neuropsychological instruments. Heinrichs \& Zakzanis (1998) carried out a large-scale comprehensive quantitative meta-analysis of cognitive impairment in schizophrenia, which involved comparisons of patients with schizophrenia $v$. controls. The greatest impairment is observed in global verbal memory functioning 8 .

Cognitive dysmetria: Andreasen et al (1998) developed a "cognitive dysmetria" model where there is a disruption in the neural circuitry involving prefrontal regions, the thalamic nuclei, and the cerebellum leads to difficulty in prioritizing, processing, coordinating, and responding to information 7 . This 'poor mental coordination' is proposed as the fundamental cognitive deficit in schizophrenia which can account for its broad diversity of symptoms.

\section{Cognitive Impairment and symptoms of} schizophrenia

Strauss (1993) hypothesised that positive symptoms in schizophrenia are related to auditory processing deficits and negative symptoms to visual/motor deficits ${ }^{26}$. Neuropsychological studies have suggested that poor performance on cognitive tests measuring primary frontal cortex functions, such as Wisconsin Card Sorting Test (WCST) trail making and measure of verbal fluency is associated with more severe negative symptoms ${ }^{27}$. While attentional deficits, thought to reflect dysfunction in more widespread neural networks are frequently associated with positive symptoms ${ }^{28}$.

Studies with different patients groups established that positive symptoms are not the sole cause of the cognitive impairment found in patients with schizophrenia. This lack of correlation was observed in first episode psychosis, chronic, as well as in elderly patients with schizophrenia ${ }^{27,29,30}$. Their findings are confirmed in the findings from CATIE schizophrenia trial9.
Baxter \& Liddle (1998) tried to integrate neuropsychology with the clinical features of schizophrenia ${ }^{31}$. Pursuing this approach to a more specific level would result in an attempt to explain specific signs or symptoms in terms of aberrant information processing. As an illustration of this approach, McKenna (1991) proposed that delusions may arise as a consequence of a dysfunctional semantic memory system ${ }^{32}$.

\section{Profiles of cognitive impairment in} schizophrenia: current status

Neuro-cognition Subcommittee for the Measurement and Treatment Research to Improve Cognition in Schizophrenia (MATRICS) project is of the opinion that the most important domains of cognitive deficit in schizophrenia are attention/vigilance, verbal learning and memory, visual learning and memory, reasoning and problem solving, speed of processing, working memory, and social cognition ${ }^{33}$.

Attention and Vigilance: Vigilance refers to the ability to maintain attention over time. Impairments in vigilance result in difficulty following social conversations and an inability to follow important instructions; simple activities such as reading or watching television become difficult ${ }^{34}$. Sustained attention or vigilance was found to be related to social problem-solving and skill acquisition.

Verbal Learning and Memory: The abilities involved in memory functioning include learning new information, retaining newly learned information over time, and recognizing previously presented material. In general, schizophrenia patients show larger deficits in new learning than in retention. Much empirical evidence points to severe verbal memory impairments in schizophrenia ${ }^{35}$. There is a clear connection between verbal memory impairments and social deficits in patients with schizophrenia, including both real-world functioning and performance on social competence tests ${ }^{34,36}$.

Visual Learning and Memory: This area of cognitive function has generally been found not to be as impaired as verbal memory ${ }^{8}$. 
Reasoning and Problem Solving: Although there are many tests of reasoning and problem solving, the most well known and most frequently used in schizophrenia research is the Wisconsin Card Sorting Test (WCST). Performance on the WCST reflects a variety of cognitive functions and is not a pure measure of executive functions ${ }^{37}$. Very poor performance of patients with schizophrenia on the WCST and the reduced activity of the dorsolateral prefrontal cortex during performance of this test led to the hypothesis of frontal lobe hypoactivation in schizophrenia ${ }^{38}$. Patients with schizophrenia who are impaired on measures of executive functions have difficulty adapting to the rapidly changing world around them.

Speed of Processing: Many neurocognitive tests like coding tasks require subjects to process information rapidly and have been found to be impaired in schizophrenia patients ${ }^{39}$. This aspect of cognitive impairment is relatively nonspecific and has been found to correlate with a variety of clinically important features of schizophrenia, such as daily life activities, job tenure and independent living status ${ }^{40-42}$.

Working Memory: Working memory has been described by various authors as a core component of the cognitive impairment in schizophrenia $^{37,43}$. It is related to functional outcome such as employment status and job tenure ${ }^{41,44}$. Neuroanatomical work has suggested that neural circuitry that includes prefrontal cortical regions mediates aspects of working memory functions. This circuitry is likely to be impaired in schizophrenia ${ }^{43,45}$.

Social Cognition: 'Theory of mind' skills and social and emotion perception and recognition have been the focus of discussion on social cognition in schizophrenia. 'Theory of mind' is the ability to infer another's intentions and/or to represent the mental states of others. Individuals with schizophrenia perform poorly on measures of 'theory of mind' abilities ${ }^{46,47}$.

\section{Cognitive impairment: 'Real-life' consequences- functional disability}

Impaired cognitive test performance in patients with schizophrenia may be argues as an epiphenomenon. So, test findings may actually reflect a lack of motivation or distraction by hallucinations. To convince sceptics that the neuropsychological impairment is important, we have to demonstrate a clear relationship between cognitive test performance and 'reallife' functional outcome. Functional capacity assessments have been developed to measure everyday living skills, social skills, vocational skills, and medication management.

Cognitive deficits in schizophrenia patients has been established as a predictor of impairment in community functioning as well as the impaired ability to perform everyday living skills ${ }^{34,40}$. Significant impact of neurocognitive impairment is seen on a patient's ability to find and maintain adequate independent living. Employment status of patients with schizophrenia affects more with impaired neurocognitive performance than clinical symptoms of schizophrenia ${ }^{48}$. Overall reductions in the quality of life are strongly associated with cognitive impairment ${ }^{49}$. Finally, cognitive deficits contribute to patterns of medication mismanagement that are associated with poor adherence and risk of relapse ${ }^{50}$. Taken together, the evidence strongly supports the view that cognitive impairment in schizophrenia is directly related to social deficits and functional outcome for many patients.

Treatment of cognitive impairment in schizophrenia: Current status

Till date no pharmacologic or behavioural treatments for cognitive impairment in schizophrenia are approved by FDA. But, many important advances are there that hold promise for the eventual development of a treatment for the same.

Effects of antipsychotic drugs on cognition: Goldberg et al (1993) reported symptomatic improvement with clozapine treatment, with no associated improvement in neuropsychological functioning51. It was concluded that 'certain cognitive deficits are relatively independent of psychotic symptoms in schizophrenia, and are probably central and enduring features of the disorder'. The effects of antipsychotic medications on cognition remain controversial. Several early studies and meta-analyses 
suggested that second-generation antipsychotic treatment may provide greater neurocognitive benefit to schizophrenia patients than firstgeneration, "typical" antipsychotics ${ }^{52}$. But CATIE study findings suggest that there were no significant benefit of four second-generation antipsychotics over first-generation antipsychotic, perphenazine ${ }^{9}$. European Union First Episode Schizophrenia Trial (EUFEST) produced similar results with no differences between treatments, even in antipsychotic-naive patients ${ }^{53}$. Overall, these data suggest that in current treatment settings, the impact of antipsychotic medications on neurocognition varies little on average, with minimal benefit for most treatments. Pharmacological augmentation as a 'cognitive enhancement strategy in schizophrenia', the treatment might include atypical antipsychotic medications, treatments for negative symptoms, and treatments for cognitive deficits.

Cognitive rehabilitation in schizophrenia: Approach to cognitive rehabilitation in schizophrenia is to try to focus on subgroups of patients who have specific cognitive abnormalities and be guided by the rehabilitation literature from brain-damaged patients. Early studies in this area focused primarily on training patients in specific neuropsychological tasks, for example, providing coaching or monetary performance incentives for performing the Wisconsin Card Sorting Test.

It was shown that patients learn better when they are prevented from making mistakes during learning 54 . Adopting this 'errorless learning' approach, O'Carroll et al (1999) showed that memory-impaired patients with schizophrenia benefited significantly from a learning approach where they were not allowed to make any mistakes during learning ${ }^{55}$.

Wykes et al (1999) conducted a randomised controlled trial of intensive cognitive remediation (targeting cognitive flexibility, working memory and planning) involving individual daily sessions for up to three months versus a control condition of intensive occupational therapy ${ }^{56}$. The cognitive remediation also included procedural and errorless learning, targeted reinforcement and massed practice. Cognitive remediation was shown to be significantly improved performance on selected tests of cognitive flexibility and memory relative to the control condition. In addition, improvements in cognitive flexibility were related to improvements in self-esteem.

\section{Indian studies on cognitive deficit in schizophrenia:}

Few Indian studies are available in relation to cognitive deficits in schizophrenia18,57,58. Most of them are replication studies of that has already been reported in western literature. Srinivasan et al (2005) reported that the neurocognitive profile of Indian patients with chronic schizophrenia resembles those of patients in developed countries ${ }^{59}$. Other Indian studies also reported patients with schizophrenia had significant deficits on multiple neuro-cognitive tests performance and negative symptoms predict poor performance in speed of processing and verbal working memory58,60,61. Impaired attentional task and Wisconsin Card Sorting Test (WCST) performances were also reported in Indian schizophrenic patient population ${ }^{58,62}$.

\section{CONCLUSION:}

We have travelled a long distance since the concept of 'dementia precox' given by Kraepelin more than a century back to modern nosological status of ICD-10 and DSM-V schizophrenia. We have moved from a middle period where cognitive impairment was not considered to be particularly important in schizophrenia, to the current view, that it may be a central and ratelimiting feature in terms of rehabilitation. But, there still lies a difficulty in detecting a consistent 'neuropsychological signature' of schizophrenia and one possible answer to that there is no such thing exist as 'schizophrenia' 25 ! Some researcher have even predicted the end of the concept of schizophrenia soon ${ }^{63}$. But we do not think we really have to be so pessimistic in our approach to understanding schizophrenia. John F. Nash Jr, the Nobel Laurette, celebrated for the originality of his thinking and his contributions to 'game theory' and pure mathematics. His schizophrenic experiences are beautifully portrayed in the book and film named "A Beautiful Mind". He once wrote, "The ideas I had about supernatural beings (delusions) came to me the same way that my mathematical ideas did. So I took them 
seriously." After years of protracting schizophrenic illness John F Nash could get back to his normal productive professional life. His life was an inspiring example that the illness does not and could not erode cognitive capabilities of all patients suffering from schizophrenia.

\section{REFERENCES:}

1. American Psychiatric Association. Diagnostic and statistical manual of mental disorders (5th ed). Washington, DC: American Psychiatric Association 2013.

2. Gaebel W, Zielasek J, P Falkai P. Psychotic Disorders in ICD-11. Downloaded from www.die-psychiatrieonline.de on 2017-01-01 | IP: 47.8.82.255.

3. Johnstone EC, Crow TJ, Frith CD, et al. Cerebral ventricular size arid cognitive impairment in chronic schizophrenia. Lancet, 1976; 11:924-926.

4. Weinberger DR, Torrey EF, Neophytide AN, Wyatt RJ. Lateral cerebral ventricular enlargement in chronic schizophrenia. Arch Gen Psychiatry 1979; 36:735-739.

5. Andreasen NC. Can Schizophrenia Be Localized in the Brain? Washington, DC: American Psychiatric Press 1986.

6. Braff DL. Information processing and attention dysfunctions in schizophrenia. Schizophr Bull 1993; 19(2):233259.

7. Andreasen NC, Paradiso S, O'Leary DS. "Cognitive Dysmetria" as an Integrative Theory of Schizophrenia: A Dysfunction in CorticalSubcortical-Cerebellar Circuitry? Schizophr Bull 1998; 24(2):203-218.

8. Heinrichs RW, Zakzanis KK. Neurocognitive deficit in schizophrenia: a quantitative review of the evidence. Neuropsychol 1998; 12:426-445.

9. Keefe RSE, Bilder RM, Harvey PD, et al. Baseline neurocognitive deficits in the CATIE schizophrenia trial. Neuropsychopharmacol 2006; 31:2033-2046.

10. Goldberg TE, Ragland JD, Torrey EF, et al. Neuropsychological assessment of monozygotic twins discordant for schizophrenia. Arch Gen Psychiatry 1990; 47:1066-1072.

11. Palmer BW, Heaton RK, Paulsen JS, et al. Is it possible to be schizophrenic yet neuropsychologically normal? Neuropsychol 1997; 11:437-446.

12. Jones P, Rodgers B, Murray R, Marmot M. Child development risk factors for adult schizophrenia in the British 1946 birth cohort. Lancet 1994; 344:1398-1402.

13. Seidman LJ, Giuliano AJ, Meyer EC, et al. Neuropsychology of the prodrome to psychosis in the NAPLS consortium: relationship to family history and conversion to psychosis. Arch Gen Psychiatry 2010; 67:578-588.
14. David AS, Malmberg A, Brandt L, et al. IQ and risk for schizophrenia: A population-based cohort study. Psychol Med 1997; 27:1311-1323.

15. Fuller $R$, Nopoulos $P$, Arndt S. Longitudinal assessment of premorbid cognitive functioning in patients with schizophrenia through examination of standardized scholastic test performance. Am J Psychiatry 2002; 159:1183-1189.

16. Reichenberg A, Caspi A, Harrington $H$, et al. Static and dynamic cognitive deficits in childhood preceding adult schizophrenia: A 30-year study. Am J Psychiatry 2010; 167:160-169.

17. Caspi A, Reichenberg A, Weiser M, et al. Cognitive performance in schizophrenia patients assessed before and following the first psychotic episode. Schizophr Res 2003; 65:87-94.

18. McCreadie RG, Latha $S$, Thara, $R$, et al. Poor memory, negative symptoms and abnormal movements in never-treated Indian patients with schizophrenia. Br J Psychiatry 1997; 171:360-363.

19. Saykin AJ, Shtasel DL, Gur RE et al. Neuropsychological deficits in neuroleptic naive patients with first-episode schizophrenia. Arch Gen Psychiatry 1994; 51:124-131.

20. Kraepelin E. Dementia Praecox and Paraphrenia. Huntingdon, NY: Robert E. Krieger Publishing 1919; Reprinted 1971.

21. Russell AJ, Munro JC, Jones PB, et al. Schizophrenia and the myth of intellectual decline. Am J Psychiatry 1997; 154:635-639.

22. Rund BR. A review of longitudinal studies of cognitive functions in schizophrenia patients. Schizophr Bull 1998; 24:425-435.

23. Carter CS, Barch DM. Cognitive neuroscience-based approaches to measuring and improving treatment effects on cognition in schizophrenia: the CNTRICS initiative. Schizophr Bull 2007; 33:1131-1137.

24. Nuechterlein KH, Green MF, Kern RS, et al. The MATRICS Consensus Cognitive Battery, part 1: test selection, reliability, and validity. Am J Psychiatry 2008; 165:203-213.

25. Blanchard JJ, Neale JM. The neuropsychological signature of schizophrenia: generalized or differential deficit? Am J Psychiatry 1994; 151:40-48.

26. Strauss ME. Relations of symptoms to cognitive deficits in schizophrenia. Schizophr Bull 1993; 19:215-231.

27. Addington J, Addington D, Maticka-Tyndale E. Cognitive functioning and positive and negative symptoms in schizophrenia. Schizophr Res 1991; 5:123-134.

28. Walker E, Harvey P. Positive and Negative Symptoms in Schizophrenia: Attentional Performance Correlates. Psychopathology 1986; 19:294-302.

29. Mohamed S, Paulsen JS, O'Leary D, et al. Generalized cognitive deficits in schizophrenia: A study of first-episode patients. Arch Gen Psychiatry 1999; 56:749-754. 
30. Davidson M, Harvey PD, Powchik P, et al. Severity of symptoms in chronically institutionalized geriatric schizophrenic patients. Am J Psychiatry 1995; 152:197-207.

31. Baxter RD, Liddle PF. Neuropsychological deficits associated with schizophrenic syndromes. Schizophr Res 1998; 30:239-249.

32. McKenna PJ. Memory, knowledge and delusions. Br J Psychiatry 1991; 159 (suppl. 14):36-41.

33. Green MF, Nuechterlein KH, Gold JM, et al. Approaching a consensus cognitive battery for clinical trials in schizophrenia: The NIMHMATRICS conference to select cognitive domains and test criteria. Biol Psychiatry 2004; 56:301-307.

34. Green MF, Kern RS, Braff DL, Mintz J. Neurocognitive deficits and functional outcome in schizophrenia: are we measuring the "right stuff"? Schizophr Bull 2000; 26:119-136.

35. Aleman A, Hijman $R$, de Haan EH, Kahn RS. Memory impairment in schizophrenia: a metaanalysis. Am J Psychiatry 1999; 156:1358-1366.

36. McClure MM, Bowie CR, Patterson TL, et al. Correlations of functional capacity and neuropsychological performance in older patients with schizophrenia: evidence for specificity of relationships? Schizophr Res 2007; 89:330-338.

37. Keefe RSE. The contribution of neuropsychology to psychiatry. Am J Psychiatry 1995; 152:6-15.

38. Goldberg TE, Weinberger DR, Berman KF, et al. Further evidence for dementia of the prefrontal type in schizophrenia? A controlled study of teaching the Wisconsin Card Sorting Test. Arch Gen Psychiatry 1987; 44:1008-1014.

39. Dickinson D, Ramsey ME, Gold JM. Overlooking the obvious: A meta-analytic comparison of digit symbol coding tasks and other cognitive measures in schizophrenia. Arch Gen Psychiatry 2007; 64:532542.

40. Evans JD, Heaton RK, Paulsen JS, et al. The relationship of neuropsychological abilities to specific domains of functional capacity in older schizophrenic patients. Biol Psychiatry 2003; 53:422-430.

41. Gold JM, Goldberg RW, McNary SW, et al. Cognitive correlates of job tenure among patients with severe mental illness. Am J Psychiatry 2002; 159:1395-1402.

42. Brekke JS, Long JD, Nesbitt N, Sobel E. The impact of service characteristics on functional outcomes from community support programs for persons with schizophrenia: a growth curve analysis. J Consult Clin Psychol 1997; 65:464-475.

43. Goldman-Rakic PS. Working memory dysfunction in schizophrenia. J Neuropsychiatry Clin Neurosci 1994; 6:348-357

44. Lysaker $P$, Bell $M$. Work rehabilitation and improvements in insight in schizophrenia. J Nero Ment Dis 1995; 183:103-106.

45. Baddeley A. Working memory. Science 1992; 255:556-559.
46. Corcoran R, Mercer G, Frith CD. Schizophrenia, symptomatology and social inference: investigating "theory of mind" in people with schizophrenia. Schizophr Res 1995; 17:5-13.

47. Drury VM, Robinson EJ, Birchwood M. Theory of mind skills during an acute episode of psychosis and following recovery. Psychol Med 1998; 28:11011112.

48. McGurk SR, Mueser KT, Harvey PD, et al. Cognitive and symptom predictors of work outcomes for clients with schizophrenia in supported employment. Psychiatr Serv 2003; 54:1129-1135.

49. Brekke JS, Kohrt B, Green MF. Neuropsychological functioning as a moderator of the relationship between psychosocial functioning and the subjective experience of self and life in schizophrenia. Schizophr Bull 2001; 27:697-708.

50. Fenton WS, Blyler CR, Heinssen RK. Determinants of medication compliance in schizophrenia: empirical and clinical findings. Schizophr Bull 1997; 23:637651.

51. Goldberg TE, Greenberg RD, Griffin SJ et al. The effect of clozapine on cognition and psychiatric symptoms in patients with schizophrenia. $\mathrm{Br} J$ Psychiatry 1993; 162:43-48.

52. Swartz MS, Perkins DO, Stroup TS, et al. Assessing clinical and functional outcomes in the Clinical Antipsychotic Trials of Intervention Effectiveness (CATIE) schizophrenia trial. Schizophr Bull 2003; 29:33-43

53. Davidson M, Galderisi S, Weiser $M$ et al. Cognitive Effects of Antipsychotic Drugs in First-Episode Schizophrenia and Schizophreniform Disorder: A Randomized, Open-Label Clinical Trial (EUFEST). Am J Psychiatry 2009; 166:675-682

54. Baddeley $A$, Wilson BA. When implicit learning fails: amnesia and the problem of error elimination. Neuropsychol 1994; 32:53-68.

55. O'Carroll RE, Russell HH, Lawrie SM, et al. Errorless learning and the cognitive rehabilitation of memory-impaired schizophrenic patients. Psychol Med 1999; 29, 105-112.

56. Wykes T, Reeder C, Corner J et al. The effects of neurocognitive remediation on executive processing in patients with schizophrenia. Schizophr Bull 1999; 25:291-307.

57. Trivedi JK, Goel D, Sharma S, et al. Cognitive functions in stable schizophrenia \& euthymic state of bipolar disorder. Ind J Med Res 2007; 126:433439.

58. Singh S, Aich TK, Bhattarai R. Wisconsin Card Sorting Test performance impairment in schizophrenia: An Indian study report. Indian Journal of Psychiatry 2017; 59 (1):88-93

59. Srinivasan L, Thara $R$, Tirupati SN. Cognitive dysfunction and associated factors in patients with chronic schizophrenia. Ind J Psychiatry 2005; 47(3): 139-143. 
60. Krishnadas $R$, Moore BP, Nayak A, Patel $R R$. Relationship of cognitive function in patients with schizophrenia in remission to disability: a crosssectional study in an Indian sample. Ann Gen Psychiatry 2007 DOI: 10.1186/1744-859X-6-19.

61. Bhattacharyya K, Guha P, Ghosal M, Barman P. Cognitive functions in schizophrenia: a comparative study in an Indian population. SL J Psychiatry 2012; 3 (1):21-27

62. Singh S, Aich TK, Ranjan S, Kumar A. Pattern of Attentional Task Impairment in Schizophrenic Patients: A Study Report. J Universal College Med Sciences. 2013; 1(2):1-7.

63. Robin M. Murray. Mistakes I Have Made in My Research Career. Schizophr Bull (2016). DOI: https://doi.org/10.1093/schbul/sbw165 